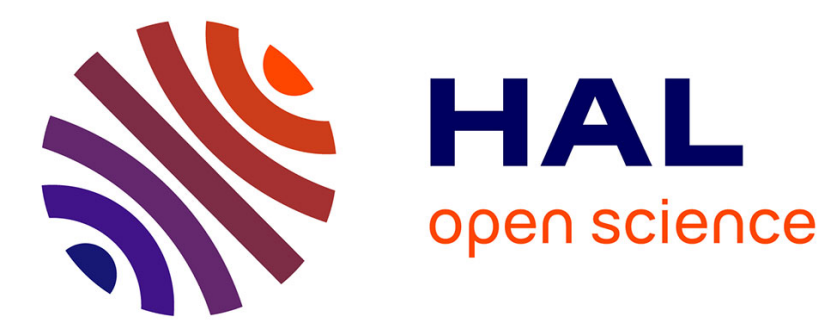

\title{
Influence de la teneur en glucides solubles des feuilles de Zea mays L. sur le choix du site de ponte de la pyrale, Ostrinia nubilalis Hbn. (Lepid. Pyralidae)
}

Vincent Fiala, Sylvie Derridj, Eugène Jolivet

\section{- To cite this version:}

Vincent Fiala, Sylvie Derridj, Eugène Jolivet. Influence de la teneur en glucides solubles des feuilles de Zea mays L. sur le choix du site de ponte de la pyrale, Ostrinia nubilalis Hbn. (Lepid. Pyralidae). Agronomie, 1985, 5 (10), pp.927-932. hal-00884723

\section{HAL Id: hal-00884723 \\ https://hal.science/hal-00884723}

Submitted on 1 Jan 1985

HAL is a multi-disciplinary open access archive for the deposit and dissemination of scientific research documents, whether they are published or not. The documents may come from teaching and research institutions in France or abroad, or from public or private research centers.
L'archive ouverte pluridisciplinaire HAL, est destinée au dépôt et à la diffusion de documents scientifiques de niveau recherche, publiés ou non, émanant des établissements d'enseignement et de recherche français ou étrangers, des laboratoires publics ou privés. 


\section{Influence de la teneur en glucides solubles des feuilles de Zea mays L. sur le choix du site de ponte de la pyrale, Ostrinia nubilalis $\mathrm{Hbn}$. (Lepid. Pyralidae)}

Vincent FIALA, Sylvie DERRIDJ $(*)$ \& Eugène JOLIVET

I.N.R.A., Laboratoire du Métabolisme et de la Nutrition des Plantes. (*) Station de Zoologie, Route de St-Cyr, F 78000 Versailles

\begin{abstract}
Le choix du site de ponte par Ostrinia nubilalis (Hbn.) varie avec l'hybride de maïs. Une tentative d'explication biochimique de ce choix est entreprise sur l'hybride le plus favorable à la ponte. Elle consiste, par passage prolongé de la plante à l'obscurité, à faire varier la teneur en glucides solubles des feuilles et à examiner les répercussions sur le comportement de l'insecte. Les femelles de pyrale pondent beaucoup moins sur les plantes appauvries et la répartition des ooplaques sur la plante est également modifiée. Une corrélation est établie entre le nombre d'ooplaques déposées sur la plante, la teneur en saccharose et, à un moindre degré, la teneur en glucose de ses feuilles.
\end{abstract}

Mots clés additionnels : Mais, saccharose, transport, effet obscurité, feuille épi, ponte, choix.
Influence of fall in soluble carbohydrates in Zea mays L. leaves on egg-laying in the corn borer (Ostrinia nubilalis Hbn. Lepid. Pyralidae).

\begin{abstract}
Egg laying by Ostrinia nubilalis ( $\mathrm{Hbn}$.) varies with the maize hybrid used. An attempt was made to provide a biochemical explanation of egg-laying site choice, using the most favourable hybrid for egg laying. Our approach consisted in modifying the soluble sugar content of leaves by prolonged dark exposure of plants and examining its effect on insect behaviour. European corn borer females laid fewer eggs on plants less rich in sugars; the distribution of egg masses on the plant was also modified. A correlation was established between the number of egg masses deposited on the plant and the sucrose (or to a lesser extent glucose) content of its leaves.
\end{abstract}

Additional key words : Sucrose, transport, dark effect, ear leaf, egg laying, choice.

\section{INTRODUCTION}

La pyrale du maïs, Ostrinia nubilalis Hbn., pond à la surface des feuilles de nombreuses espèces de plantes supérieures. Chez le maïs elle occasionne de gros dégâts aux cultures; toutefois les pontes déposées par l'insecte varient avec l'hybride (ANGLADE $\boldsymbol{e t}$ al., 1980). Aussi l'intérêt s'est-il porté récemment sur l'étude des facteurs qui déterminent le choix de la femelle à pondre plutôt sur une plante que sur une autre et, pour une plante donnée, à déposer ses œufs plutôt sur une feuille que sur une autre (DERRIDJ \& FIALA, 1983).

Dans le passé, PATCH (1942) avait rattaché ce choix à la hauteur du maïs puis BURGSTALLER (1974) avait abordé l'étude de facteurs endogènes et exogènes sur la sensibilité des génotypes de maïs au dépôt de ponte de la pyrale. Plus récemment, Derridj \& Fiala (1983) ont montré que, parmi ces facteurs endogènes, la teneur en glucides solubles de la feuille semblait avoir son importance, les pontes étant plus fréquentes sur l'hybride expérimental $\mathrm{A} \times \mathrm{F}$, plus riche en ces composés, que sur la variété «LG11», à teneur plus faible.

Pour vérifier cette relation entre la teneur en glucides de la feuille et le dépôt des pontes, nous avons utilisé un seul hybride dont les teneurs des feuilles en glucides solubles sont soumises à des variations sous l'influence de facteurs externes. On peut ainsi exclure, comme facteurs intervenant dans le choix de l'insecte, de nombreux paramètres inconnus liés aux caractères 
génétiques des hybrides et/ou aux caractères phénologiques.

Deux possibilités s'offraient à nous : soit enrichir en glucides des feuilles détachées en les trempant dans des solutions de saccharose, soit abaisser le taux de glucides dans la plante entière en la soumettant à une obscurité prolongée (ESCHRICH \& BURCHARDT, 1982). Nous avons choisi la $2^{\circ}$ approche, plus naturelle. La présente note rapporte donc les effets d'une diminution de la teneur en glucides des feuilles de maïs provoquée par l'obscurité prolongée sur le nombre de pontes de la pyrale.

\section{MATÉRIEL ET MÉTHODES}

\section{A. Matériel végétal}

Le maïs, hybride simple $A \times F$, choisi parce que riche en glucides solubles et favorable à la ponte de la pyrale, est cultivé en serre dans des conteneurs de 71 (1 plante par conteneur), sur vermiculite, en conditions hydroponiques, jusqu'au début de la floraison mâle (juin 1983). La photopériode est celle de la saison, les températures jour/nuit avoisinent $25^{\circ} \mathrm{C} /$ $17^{\circ} \mathrm{C}$ et l'état hygrométrique $70 \mathrm{p} .100$.

\section{B. Obtention des pyrales}

Les pyrales proviennent de chenilles recueillies dans des tiges de maïs, l'automne précédent, dans des champs de la Beauce $(100 \mathrm{~km}$ environ à l'Ouest de Paris). Les larves sont laissées dans les tiges conservées à l'extérieur jusqu'au mois d'avril de l'année suivante, date à partir de laquelle elles sont mises à $25 \pm 2{ }^{\circ} \mathrm{C}$ sous une photopériode de $16 \mathrm{~h}$ et une humidité relative de 80 p. 100 . Les chrysalides au fur et à mesure de leur formation sont placées à des températures pouvant varier de 15 à $25^{\circ} \mathrm{C}$ afin de faire coïncider le plus possible les émergences des adultes.

Les adultes sont mis dans des cagettes de $180 \mathrm{~mm}$ de diamètre sur $200 \mathrm{~mm}$ de hauteur, à raison de 5 femelles et 5 mâles par cagette. L'ensemble est placé dans une chambre climatisée à $25 \pm 2{ }^{\circ} \mathrm{C}, 16 \mathrm{~h}$ de photopériode et 70 p. 100 d'hygrométrie.

\section{Méthodes expérimentales}

\section{Expérience préliminaire en l'absence de pyrale}

Cette expérience est effectuée afin de déterminer comment varient les glucides solubles de la feuille de mais sous l'effet d'une obscurité prolongée. Trois lots de plantes cultivés en serre, en conteneurs, sont donc préparés (fig. 1). Un $1^{\text {er }}$ lot témoin (T) est gardé en serre sous la photopériode naturelle de la saison : jour de $5 \mathrm{~h}$ à $20 \mathrm{~h}$, nuit de $20 \mathrm{~h}$ à $5 \mathrm{~h}$ (heure universelle). Un $2^{\mathrm{e}}$ lot (obsc. 1) est placé à partir de $12 \mathrm{~h}$, pendant $6 \mathrm{~h}$, à l'obscurité dans une chambre climatisée $\left(15^{\circ} \mathrm{C}\right)$; il est remis en serre à partir de $18 \mathrm{~h}$ le même jour. Un $3^{\mathrm{e}}$ lot (obsc. 2) est placé à partir de $5 \mathrm{~h}$, pendant $15 \mathrm{~h}$, à l'obscurité dans la même chambre climatisée que le lot obsc. 1 ; il est remis en serre à $20 \mathrm{~h}$. Ces traitements ne sont effectués qu'une fois (fig. 1).

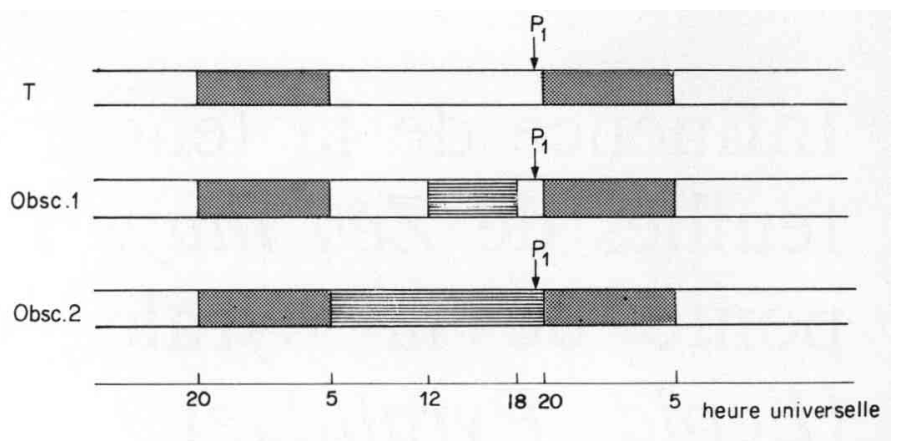

Figure 1

Représentation schématique du régime lumineux appliqué aux plantes de maïs (expérience en l'absence de pyrale). $T=$ témoin, Obsc. 1 = obscurité intermittente; Obsc. 2 = obscurité continue; $P 1=$ prélèvement des feuilles pour analyses des glucides.

Lumière naturelle; ; Obscurité naturelle; apportée.

Schematic representation of light regimes applied to maize (without insect). $T=$ control; Obsc. 1 = intermittent dark; Obsc. $2=$ continuous dark ; PI = sampling of leaves for carbohydrate analysis.

Des prélèvements pour analyses de glucides sont effectués à $19 \mathrm{~h}$ (P1), sur les feuilles situées à 3 niveaux différents de la plante : la $2^{\mathrm{c}}$ feuille audessus de l'épi $(\mathrm{E} 0+2)$, la feuille de l'épi (E0), la $2^{e}$ feuille en dessous de l'épi $(\mathrm{E} 0-2)$. Chaque échantillon comprend 2 feuilles et on prélève sur chaque feuille un segment de longueur $50 \mathrm{~mm}$, situé à une distance de la base de la feuille comprise entre 100 et $150 \mathrm{~mm}$. Les prélèvements de feuilles et les analyses de glucides sont répétés 4 fois.

\section{Expérience en présence de pyrale}

L'expérience porte seulement sur les plantes $\mathrm{T}$ et obsc. 2 (fig. 2) maintenues en serre en conteneurs, eux-mêmes placés dans 4 cages cubiques mobiles de $2 \mathrm{~m}$ d'arête, recouvertes d'un voilage en tergal blanc.

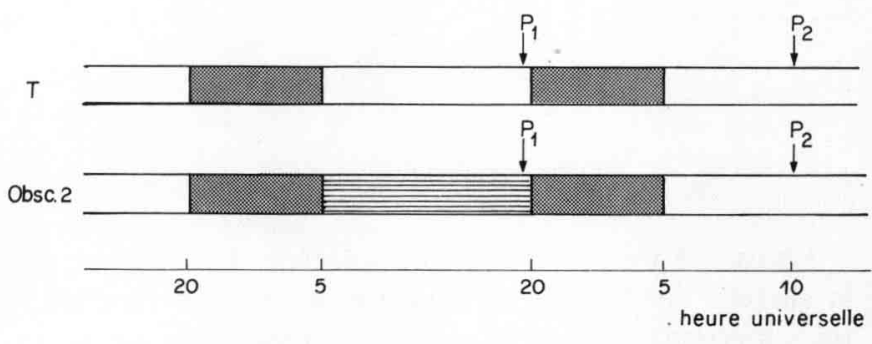

Figure 2

Représentation schématique du régime lumineux appliqué aux plantes de maïs (expérience en présence de pyrale). $T=$ témoin, Obsc. 2 = obscurité continue; $P I=$ prélèvement des feuilles pour analyses des glucides ; $P 2=$ prélèvement des feuilles pour analyses des glucides et dénombrement des pontes de la pyrale.

Schematic representation of light regimes applied to maize (with insect). $T=$ control ; Obsc. $2=$ continuous dark; $P I=$ sampling of leaves for carbohydrate analysis; $P 2=$ sampling of leaves for carbohydrate analysis and counting of the number of egg masses. 
Chacune de ces cages contient 3 rangs de 6 plantes, alternées par lot ( $\mathrm{T}$, obsc. 2) sur le rang et d'un rang à l'autre, de manière que les feuilles se trouvent en majorité dans le plan perpendiculaire à l'axe des rangs et qu'il y ait choix pour l'insecte dans les 2 directions suivant le rang et perpendiculairement au rang (fig. 3).

$72 \mathrm{~h}$ après leur émergence, alors que les femelles pondaient depuis $48 \mathrm{~h}$, les adultes sont introduits dans les cages, dans la serre, où on venait de placer les maïs. Le comportement de dépôt des ooplaques étant crépusculaire, les insectes sont lâchés le soir à $19 \mathrm{~h}$ dans les cages placées dans la serre ( 25 femelles et 25 mâles par cage). Au même moment, à $19 \mathrm{~h}$, sur des plantes homologues à celles utilisées pour l'expérimentation sur insectes, on effectue un prélèvement $\mathrm{P} 1$ de feuilles pour l'analyse des glucides (fig. 2). Un $2^{\mathrm{e}}$ prélèvement P2 est effectué le matin suivant, à $10 \mathrm{~h}$, après un éclairement naturel de $5 \mathrm{~h}$, sur les plantes des 2 lots $\mathrm{T}$ et obsc. 2 afin de connaître l'effet de cet éclairement sur l'évolution des glucides des 2 lots.

Le dénombrement des pontes a lieu au prélèvement P2. Le test $\chi 2$ est appliqué aux moyennes des nombres de pontes notés dans chaque cage pour les niveaux foliaires considérés.

\section{Analyse des glucides}

Aussitôt après leur prélèvement les feuilles sont découpées en segments immédiatement fixés dans l'éthanol à 80 p. 100 et l'ensemble est conservé à $4{ }^{\circ} \mathrm{C}$. Les extractions puis les dosages des glucides solubles par une méthode enzymatique (BERGMEYER, 1979) sont effectués au plus tard $48 \mathrm{~h}$ après fixation des tissus (DERRIDJ \& FIALA, 1983). Un contrôle de la composition en glucides des feuilles est réalisé par chromatographie liquide sur analyseur Biotronik (VOELTER \& BAUER, 1976 ; FIALA, 1983).

\section{RÉSULTATS}

\section{A. Expérience en l'absence de pyrale : composition en glucides solubles des feuilles de maïs en relation avec leur position sur la tige et la durée de la période d'obscurité}

Dans le lot T, les glucides solubles (saccharose, glucose, fructose) des feuilles se répartissent selon un gradient décroissant de l'étage $\mathrm{E} 0+2$ à l'étage $\mathrm{E} 0-2$ (tabl. 1). La majeure partie de ces glucides solubles est représentée par le saccharose.

Si on soumet les plantes de $12 \mathrm{~h}$ à $18 \mathrm{~h}$ (fig. 1) à une obscurité supplémentaire (obsc. 1), les teneurs en glucides solubles totaux et notamment celles en saccharose, diminuent nettement et le gradient n'existe plus (tabl. 1). L'effet de l'obscurité est observé surtout au niveau de l'étage supérieur $(\mathrm{E} 0+2)$ et, à un moindre degré, au niveau de la feuille de l'épi E0.

Si on maintient les plantes dans une obscurité continue (fig. 1) de $24 \mathrm{~h}$ (obsc. 2), les teneurs en glucides solubles totaux et en particulier celles en saccharose baissent encore plus fortement, même au niveau de la feuille de l'épi (E0); le gradient tend à s'inverser (tabl. 1). Sous l'effet de ce traitement, les teneurs en glucides solubles, au niveau de la feuille E0 +2 , ne

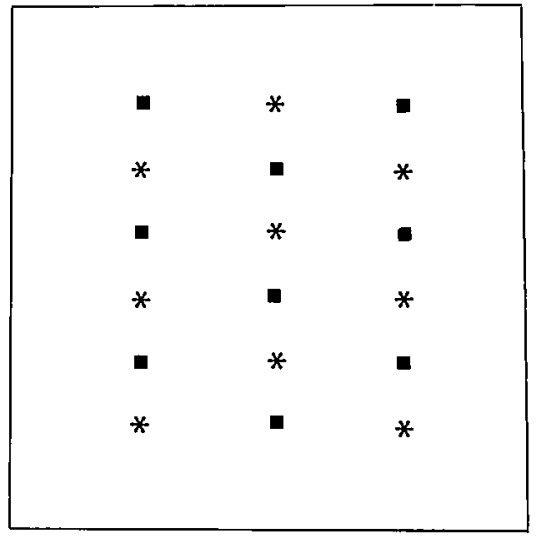

Figure 3

Expérimentation du choix en présence de l'insecte : disposition des plantes des lots $T$ ( $\star$ ) et obsc. 2 ( $\mathbf{0}$ ) dans une cage cubique de $8 \mathrm{~m}^{3}$.

Prefered experimental lay-out in the presence of the insect : arrangement of the plants $T(\star)$ and obsc. $2(\mathbf{0})$ in a cubic cage of $8 \mathrm{~m}^{3}$.

représentent plus que 20,4 p. 100 pour les glucides totaux et 12,1 p. 100 pour le saccharose par rapport à celles du témoin.

\section{B. Expérience en présence de pyrale : mise en évi- dence d'une relation entre le dépôt de ponte de la pyrale et les teneurs en glucides solubles des feuil- les des plantes témoins et des plantes mises en obs- curité continue}

Compte tenu des résultats de l'expérience précédente, nous avons choisi de mettre seulement les plantes en obscurité continue (obsc. 2), traitement qui appauvrit les plantes d'une manière plus prononcée. Nous avons limité les analyses de glucides solubles à un seul niveau foliaire, celui de la feuille de l'épi (E0) sur laquelle est observé le plus grand nombre de pontes. Il est probable que l'écart observé dans les teneurs en glucides entre les 2 lots persiste pendant toute la période nocturne.

Le dénombrement des pontes au prélèvement P2 (fig. 2), effectué sur les 5 feuilles des étages E0 +2 à EO - 2 où se trouve la majorité des pontes, montre une diminution de leur nombre sur les plantes obsc. 2 , appauvries en glucides (tabl. 2). Sur les plantes entières rapportées à 100 , pour la totalité des niveaux, on dénombre $127,7 \pm 31,2$ pontes pour 100 plantes témoins et $44,3 \pm 23,7$ sur 100 plantes obsc. 2. La somme des $\chi 2$ calculés pour chaque cage $(=10,34)$ montre qu'il existe une différence hautement significative au seuil de 2,5 p. 100 entre ces 2 lots.

Il est intéressant aussi de constater que la répartition des pontes sur les niveaux foliaires entre $\mathrm{E} 0+2$ et E0 - 2 est modifiée par la diminution de la teneur en sucres solubles (fig. 4). En effet, sur les plantes obsc. 2 , il n'y a plus de maximum de pontes au niveau de la feuille de l'épi contrairement à ce qui est observé sur les plantes $T$. Le test $\chi 2$ montre que les répartitions des pontes à ces niveaux foliaires, sur les 2 types de plantes, sont significativement différentes au seuil de 5 p. $100(\chi 2=4,42)$. 
TABLEAU 1

Teneurs en glucides solubles ( $\mathrm{mg} / \mathrm{g} M F$ ) des feuilles de maïs lors du prélèvement PI en fonction du niveau foliaire des plantes soumises aux 3 traitements lumineux.

Soluble sugar content of maize leaves at sampling PI as a function of leaf stratum of plants exposed to three light treatments.

\begin{tabular}{|c|c|c|c|c|c|}
\hline Traitement & $\begin{array}{c}\text { Position } \\
\text { de la feuille }\end{array}$ & Saccharose & Fructose & Glucose & Total \\
\hline Témoin & $\begin{array}{l}\mathrm{E} 0+2 \\
\mathrm{E} 0 \\
\mathrm{E} 0-2\end{array}$ & $\begin{array}{r}10,7 \\
8,5 \\
7,4\end{array}$ & $\begin{array}{l}1,5 \\
1,3 \\
0,6\end{array}$ & $\begin{array}{l}2,0 \\
1,3 \\
0,9\end{array}$ & $\begin{array}{r}14,2 \\
11,1 \\
8,9\end{array}$ \\
\hline Obsc. 1 & $\begin{array}{l}\mathrm{E} 0+2 \\
\mathrm{E} 0 \\
\mathrm{E} 0-2\end{array}$ & $\begin{array}{l}5,8 \\
5,5 \\
5,9\end{array}$ & $\begin{array}{l}1,1 \\
1,0 \\
0,9\end{array}$ & $\begin{array}{l}1,0 \\
1,3 \\
1,1\end{array}$ & $\begin{array}{l}7,9 \\
7,8 \\
7,9\end{array}$ \\
\hline Obsc. 2 & $\begin{array}{l}\mathrm{E} 0+2 \\
\mathrm{E} 0 \\
\mathrm{E} 0-2\end{array}$ & $\begin{array}{l}1,3 \\
2,0 \\
2,4\end{array}$ & $\begin{array}{l}0,7 \\
0,8 \\
0,8\end{array}$ & $\begin{array}{l}0,9 \\
0,8 \\
0,7\end{array}$ & $\begin{array}{l}2,9 \\
3,6 \\
3,9\end{array}$ \\
\hline
\end{tabular}

TABLEAU 2

Composition en glucides solubles ( $\mathrm{mg} / \mathrm{g} \mathrm{MF}$ ) de la feuille de l'épi (EO)

lors des prélèvements $P 1$ et $P 2$ de plantes soumises à 2 traitements lumineux.

Soluble sugar content of ear leaf (E0) at sampling PI and P2 of plants exposed to two light treatments.

\begin{tabular}{|c|c|c|c|c|c|c|c|}
\hline & \multicolumn{3}{|c|}{ Prélèvement Pl } & \multicolumn{3}{|c|}{ Prélèvement $\mathrm{P} 2$} & \multirow{2}{*}{$\begin{array}{c}\text { Dénombrement } \mathrm{P} 2 \\
\text { Nombre de pontes } \\
\text { pour } 100 \text { plantes }\end{array}$} \\
\hline & saccharose & fructose & glucose & saccharose & fructose & glucose & \\
\hline Témoin & 16,7 & 9,6 & 17,1 & 30,0 & 9,2 & 18,7 & $83,3 \pm 36,3$ \\
\hline Obsc. 2 & 8,1 & 9,4 & 14,6 & 31,4 & 9,2 & 13,5 & $22,0 \pm 9,4$ \\
\hline t ou $\chi^{2}$ & 3,96 & 0,13 & 1,00 & 0,77 & 0,05 & 2,01 & 9,5 \\
\hline Signification (l) & +++ & NS & NS & NS & NS & NS & ++ \\
\hline
\end{tabular}

(1) Niveau de signification du test de la différence entre les 2 lots : NS : non significatif au niveau 5 p. $100 ;$ insignificant at $5 \%$ level. ++ significatif au niveau 2,5 p. 100 ; significant at $2.5 \%$ level. +++ : significatif au niveau 1 p. 100 ; significant at $1 \%$ level. $t$ : test pour le dosage des glucides ; test for sugar analysis. $\chi^{2}$ : test pour le nombre de pontes ; test for number of egg masses.

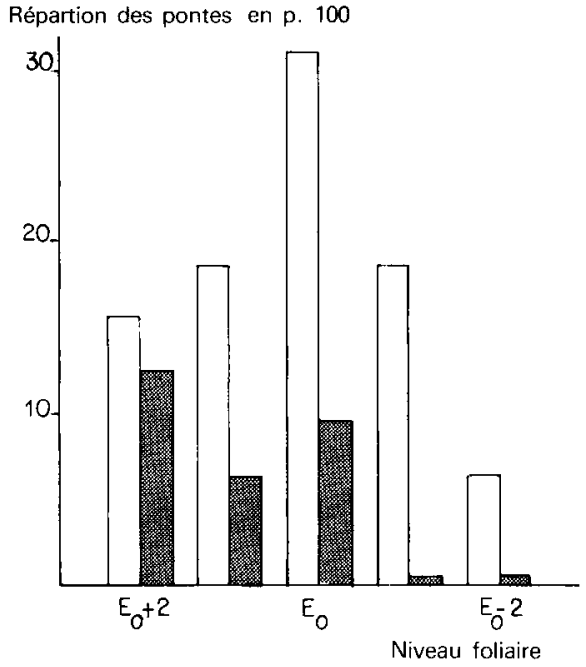

Figure 4

Répartition des pontes de la pyrale sur les étages foliaires compris entre EO +2 et EO - 2 pour le relevé P2.

Distribution of corn borer egg masses according to leaf stratum including EO +2 and EO - 2 for sampling $P 2$.

\section{DISCUSSION}

Nos résultats concernant les variations des teneurs en glucides solubles totaux (saccharose + fructose + glucose) dans les feuilles de maïs en fonction du niveau de l'étage foliaire sont en accord avec ceux de CARLES et al. (1953), EASTIN (1970), FAIREY \& DAYNARD (1978). Ils montrent en outre que c'est surtout le saccharose qui est la cause principale de ces variations. La richesse plus élevée en glucides solubles des feuilles au-dessus de l'épi est liée à leur plus grande activité photosynthétique à ce stade phénologique (PRIOUL \& ROCHER, 1983). Il n'est pas exclu que d'autres composés solubles, acides organiques ou acides aminés libres, qui sont également soumis à variations sous l'action de l'obscurité, puissent intervenir dans le comportement de la pyrale.

Chez des plantes placées dans des conditions normales comme celles du lot témoin, le saccharose est le glucide le plus répandu parmi les glucides solubles et il est pratiquement le seul glucide transporté par la sève élaborée. Dans les feuilles de maïs, plante de type $\mathrm{C} 4$, le transport du saccharose provenant de la photosynthèse se fait sur une courte distance puisque les cel- 
lules synthétisant le saccharose sont celles de la gaine périvasculaire (PRIOUL \& ROCHER, 1983). Le saccharose entre dans l'espace libre (apoplaste) : une partie est transportée à longue distance dans cet espace sans être hydrolysé (HEYSER et al., 1977) et une autre est véhiculée activement dans le phloème. Le saccharose est pratiquement le seul glucide présent dans l'espace libre, les teneurs en fructose et en glucose étant beaucoup plus faibles. Les teneurs en saccharose apoplastique (paroi cellulaire + espace intercellulaire) varient au cours de la période de $24 \mathrm{~h}$ et le rapport saccharose apoplastique/saccharose symplastique (ensemble des protoplasmes reliés par plasmodesmes) est significativement plus élevé à la fin de la période diurne (DELROT et al., 1983). Le saccharose apoplastique serait transporté vers les cellules épidermiques. Cette répartition dans les tissus foliaires pourrait avoir une grande importance au niveau de la perception sensorielle par la pyrale qui se trouverait ainsi en contact avec les sucres.

Les plantes de maïs placées sous une période d'obscurité continue de $24 \mathrm{~h}$ réagissent par une baisse de leurs teneurs en saccharose (tabl. 1 et 2 ) et vraisemblablement en saccharose apoplastique. Cette baisse est temporaire, car si, après ces $24 \mathrm{~h}$ d'obscurité, les plantes sont soumises à une photopériode naturelle, les différences en glucides solubles entre le lot témoin (T) et le lot obscurité (obsc. 2) disparaissent. Les analyses effectuées sur les feuilles du prélèvement $\mathrm{P} 2$ après séjour des plantes pendant $5 \mathrm{~h}$ à la lumière le montrent bien (tabl. 2). Sur les plantes de maïs appauvries, le nombre de pontes déposées par la pyrale est significativement plus faible. Il apparaît donc un parallélisme entre les variations de teneurs en saccharose, le choix des sites de pontes et l'importance de ces pontes. Nous avons observé en outre que les pontes se trouvent souvent à proximité des nervures qui sont les sites principaux d'accumulation du saccharose (LÉGER et al., 1982). Ce fait peut souligner encore l'existence d'une corrélation entre la teneur en saccharose et le dépôt des pontes de l'insecte.

\section{CONCLUSION}

Les femelles de pyrale choisissent toujours entre 2 plantes, à un niveau foliaire donné, la feuille la plus riche en sucres. Toutefois les insectes ne " sélectionnent » pas la feuille la plus riche d'une plante. Le choix se fait plutôt sur une strate horizontale d'une plante à l'autre, que verticalement d'une feuille à l'autre sur la même plante. Au stade phénologique choisi (floraison mâle), la majorité des pontes se situe dans la zone de l'épi : il n'est pas exclu que celui-ci ait aussi un rôle dans le choix du site de ponte. CANTELO \& JACOBSON (1979) ont montré l'effet attractif de certaines substances volatiles des soies de l'épi de maïs sur la pyrale.

Le rôle des substances nutritionnelles dans le dépôt des pontes des lépidoptères non nectarivores n'a pas encore été signalé. Les sucres solubles interviendraient comme substances allélochimiques plutôt que par leurs qualités nutritionnelles. La pyrale qui est polyphage possède probablement des chimio-récepteurs peu spécifiques. Les critères de reconnaissance sont peut-être multiples et les sucres pourraient être perçus directement ou indirectement par l'insecte, associés ou non à d'autres caractères. Les sucres interviendraient comme une kairomone bénéfique pour la descendance. Les chenilles de pyrale ont en effet un "saccharotropisme » évident (BATCHELDER, 1949 ; BECK, $1956 a, 1956 b)$. En outre, RASSIPOUR (1979) a montré l'effet phagostimulant des sucres sur les jeunes chenilles. D'autres expériences que nous poursuivons actuellement devraient approfondir les relations que nous venons de montrer entre la teneur en glucides solubles, notamment en saccharose de la feuille, et le dépôt de pontes de la pyrale.

Reçu le 12 février 1985. Accepté le 25 juillet 1985.

\section{REMERCIEMENTS}

Ce travail a bénéficié de la collaboration de M. Augendre et Y. DURAND (Station de Zoologie), ainsi que de celle de M. MARTIN (Laboratoire du Métabolisme et de la Nutrition des Plantes). Nous leur adressons nos vifs remerciements.

\section{RÉFÉRENCES BIBLIOGRAPHIQUES}

Anglade P., Derridj S., Durand Y., 1980. First observations upon the preference of the European corn borer and their significance in breeding for resistance. 2nd Eucarpia/I.O.B.C. Meeting. Bull. S.R.O.P., 4, 105-108.

Batchelder C. H., 1949. European corn borer location on the corn plant as related to insecticidal control. U.S. Dep. Agric, tech. Bull, 976, $1-20$.

Beck S. D., 1956a. Nutrition of the European corn borer, Pyrausta nubilalis Hbn. IV. Feeding reactions of first instar larvae. Ann. Entomol. Soc. Am., 49, 399-405.

Beck S. D., 1956b. The European corn borer, Pyrausta nubilatis $\mathrm{Hbn}$. and its original host plant. I. Orientation and feeding behaviour of the larva on the corn plant. Ann. Entomol. Soc. Am., 49, $552-558$

Bergmeyer H. U., 1979. Principes de l'analyse enzymatique. Tech nique et Documentation, Paris, 303 p.

Burgstaller H., 1974. Untersuchungen über den Einflüss endogener und exogener Faktoren auf die Anfälligkeit von Maïgenotypen gegenüber den Maiszünsler $\mathrm{O}$. nubilalis (Hbn.). These-Agrarbiologie, Univ. Hohenheim (L.H), $154 \mathrm{p}$.
Cantelo W. W., Jacobson M., 1979. Corn silk volatiles attract many pest species of moths. J. environ. Sci. Health, A 14, 8, 695 707

Carles J., Soubies L., Gadet R., 1953. La migration des glucides vers les grains de maïs. C. $R$. Acad. Sci., Paris Ser. D., 236, 1814 1816

Delrot S., Faucher M., Bonnemain J. L., Bonmort J., 1983 Nycthemeral changes in intracellular and apoplastic sugars in Vicia faba leaves. Physiol. Vég., 21, 459-467.

Derridj S., Fiala V., 1983. Sucres solubles des feuilles de mais (Zea mays L.) et oviposition de la pyrale (Ostrinia mubilalis $\mathrm{Hbn}$.). C. $R$. Acad. Agric. Fr., 69, 465-472.

Eastin J. A., 1970. C-14 labeled photosynthate export from fully expanded corn (Zea mays L.) leaf tlades. Crop Sci., 10, 415-418.

Eschrich W., Burchardt R., 1982. Reaction of phloem export in mature maize leaves after a dark period. Planta, 155, 444-448.

Fairey N. A., Daynard T. B., 1978. Quantitative distribution of assimilates in component organs of maize during reproductive growth. Can. J. Plant Sci., 58, 709-717, 
Fiala V., 1983. Approche biochimique du forçage de la racine de chicorée Witloof (Cichorium intybus L.) par l'étude des variations de sa composition en gluciaies. I hese Doct. Ing., Univ. Paris VI, $81 \mathrm{p}$.

Heyser W., Heyser R., Eschrich W., Fritz E., 1977. The influence of externally supplied sucrose on phloem transport in the maize leaf strip. Planta, 137, 145-151.

Léger A., Delrot S., Bonnemain J. L., 1982. Properties of sugar uptake by wheat leaf fragments : effects of ageing and $\mathrm{pH}$ dependence. Physiol. Vég., 20, 651-659.

Patch L. H., 1942. Height of corn as a factor in egg laying by the European corn borer moth in the one generation area. J. agric. Res., 64, 503-515.
Prioul J. L., Rocher J. P., 1983. Les transports d'assimilats chez le mais : mécanisme, rôle des facteurs externes, application à des comparaisons intergénotypiques. Coll. « Physiologie du Maïs », Royan (France), 39 p.

Rassipour A., 1979. Contribution à l'étude de la nutrition glucidique de la pyrale du maïs Ostrinia nubilalis $\mathrm{Hbn}$. Lepidoptera Pyralidae. Thèse Doct. Ing., Paris XI, $117 \mathrm{p}$.

Voelter W., Bauer H., 1976. High-performance liquid chromatography analysis of the isomerization products of carbohydrates. J. Chromalogr., 126, 693-703. 